\title{
por una nueva \\ institución educativa
}

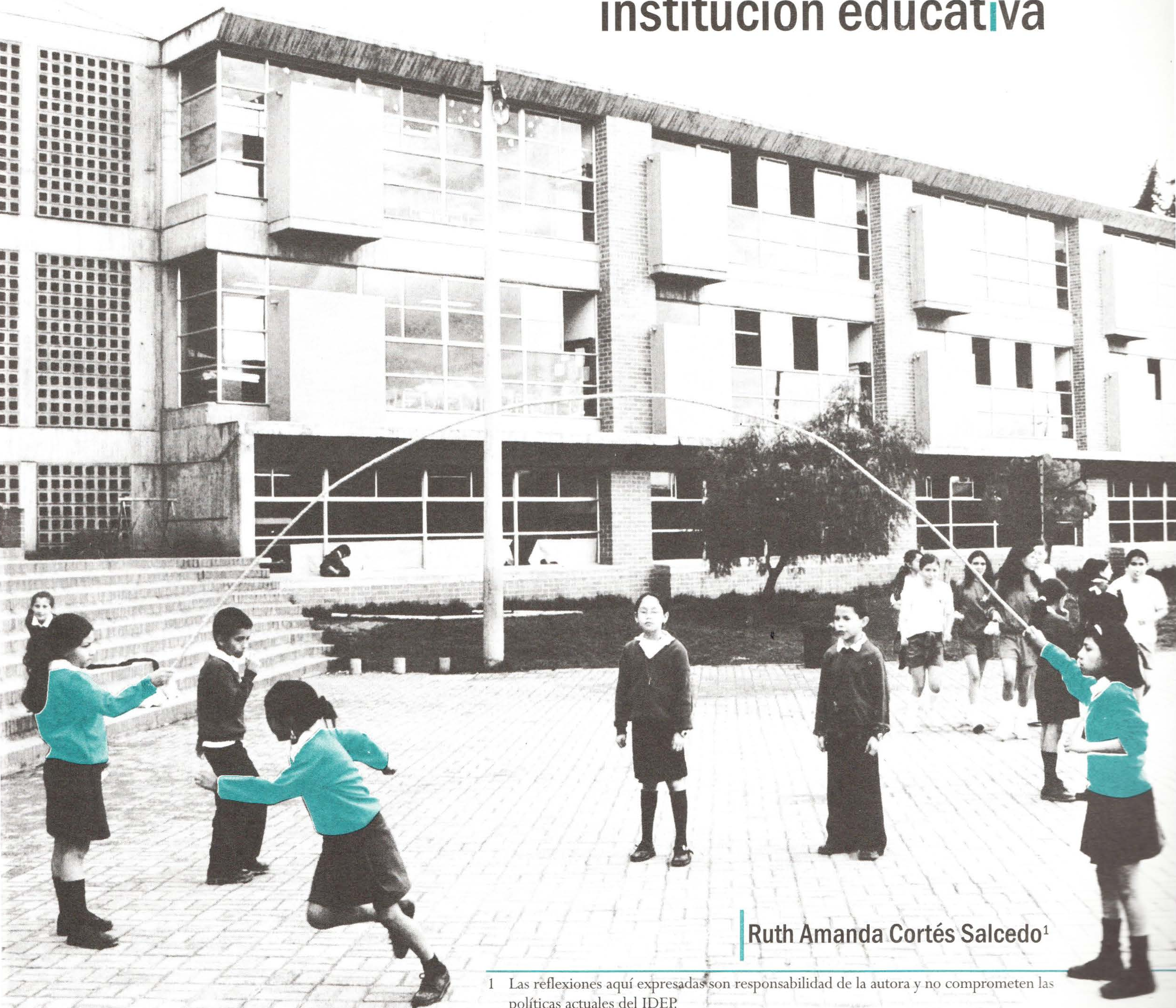
políticas actuales del IDEP. 


\section{Ruth Amanda Cortés}

es Licenciada en Ciencias sociales de la Universidad Pedagógica Nacional, especialista en enseñanza de la historia y magíster en Educación y Desarrollo Social, estudiante de doctorado en Educación. Profesora de educación básica y universitaria; Coordinadora del Programa de Formación Permanente de Docentes en la Fundación Universitaria Los Libertadores y del Proyecto Jóvenes Tejedores de Sociedad en el Departamento Administrativo de Acción Comunal Distrital. Asesora del Área de Comunicación Educativa, Subdirectora Académica del IDEP; actualmente se desempeña como profesional del Área Académica de la misma Institución.

Correo electrónico: amandacortes2006@yahoo.es

\section{Resumen}

El presente ensayo presenta una reflexión en torno al concepto de política pública educativa desde varias ópticas. Primera, desde el punto de vista conceptual asumiendo una definición de política pública que permita transitar por el concepto de la educación en sí misma. En segundo lugar, elabora un análisis de la política educativa centrada en el tema de la calidad y en el significado de la evaluación en ese contexto; por último, aborda el análisis a partir de la caracterización del surgimiento de una nueva institucionalidad educativa.

\section{Palabras clave: Política pública, Calidad de la educación, Evaluación, Institución educativa Abstract \\ This essay explores the concept of public and government policy in education from different perspectives. First, it assumes a conceptual framework from education itself; secondly, the author prepares an analysis of the policy of education according to the notion of quality. Finally, the analysis is performed according a characterization of a new educational institution.}

\section{Key words: Public policy, Quality of education, Evaluation, Schools}




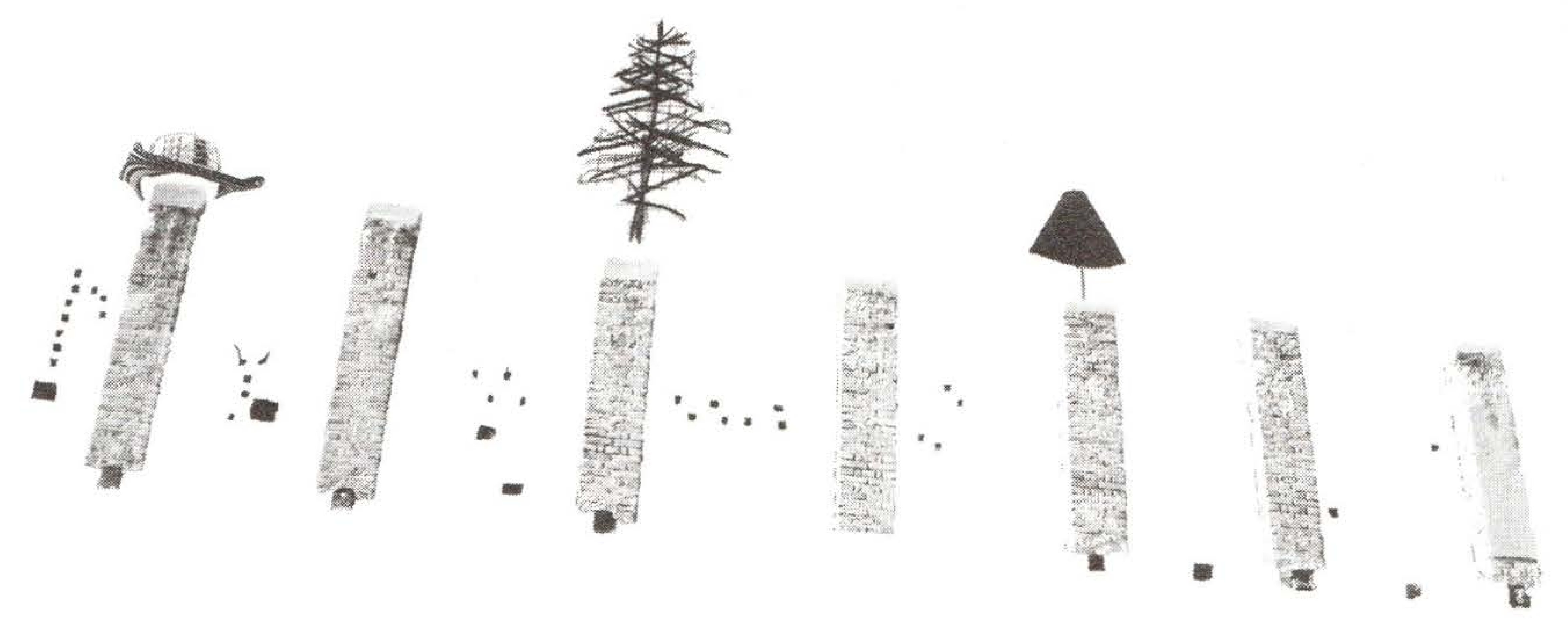

\section{¿A qué nos referimos cuando hablamos de política pública?}

La noción de política pública se compone de dos enunciados: la política y lo público, sin mayores pretensiones que la de hacer algunas precisiones sobre el asunto se retoma a Henry Giroux $(2001,49)$ cuando afirma que "La política es el registro resultante de la acción moral, el marco de la sociedad civilizada que evita que la justicia y la compasión mueran en cada uno de nosotros, a sí como una llamada para reconocer la exigencia de la humanidad por erradicar el sufrimiento innecesario y a la vez reafirmar, la libertad, la igualdad y la esperanza"

A la política se le pueden señalar tres propiedades pragmáticas: i) el escenario desde y donde se gobierna, ii) como disposición para la organización, la movilización y lucha por el gobierno y el poder; y iii) como despliegue de finalidades y programas de las autoridades públicas para efectos de las acciones de gobierno en un sector de la sociedad, en un territorio y dirigidas a obtener unos determinados objetivos. (SED - IDEP: 2004)
Para el segundo enunciado se acude a la definición de esfera pública de Fernando Bárcena $(1995,3)$ que la concreta como: "El ámbito de revelación en el que reinan la libertad y la igualdad, el espacio en el que los individuos, en tanto que ciudadanos, interactúan por medio del habla y la persuasión, y el contexto propicio en el que muestran sus genuinas identidades para decidir, mediante la deliberación colectiva, asuntos de interés común"

Rawls, citado por Francisco Reyes, $(2002,14)$ define dos esferas de la vida del hombre, una esfera pública en la que está el mundo de la ciudadanía y una esfera privada, a la que pertenecen las creencias y convicciones personales. En la distinción que hace Rawls de las esferas de la vida del individuo, le otorga a éste la capacidad de discernimiento en dos planos: en la esfera privada es racional, es decir, la capacidad que tiene de definir sus intereses, la noción del bien y de la vida buena desde una ética de las convicciones y en la esfera de lo público el individuo es razonable en tanto está en capacidad de dialogar con los intereses de los otros y encontrar acuerdos y consensos mínimos entre ellos que les permita la coexistencia. 
Desde estas dos posiciones, la política pública se definiría como una acción moral que regula la justicia y dignifica la condición humana, materializándose en un mecanismo organizador y catalizador de esas acciones, producto de decisiones deliberadas colectivamente con miras a atender intereses comunes de una sociedad. Sería también una forma de garantizar los derechos, fomentar el ejercicio de la ciudadanía y profundizar la democracia.

En suma, la política pública es

"el conjunto coherente de principios, objetivos, estrategias y planes de acción que identifican, comprenden y abordan las problemáticas de una sociedad (económicas, políticas, sociales, culturales o ambientales) o condiciones de un grupo poblacional o comunidad, con el fin de darles solución o mejorar las condiciones de vida a partir de la acción colectiva, en el marco de un proyecto democrático de sociedad"',

o "... un conjunto de decisiones que se traducen en acciones, estratégicamente seleccionadas (dentro de un conjunto de alternativas, conforme a una jerarquía de valores y preferencia de los interesados). Su dimensión es pública por el tamaño del agregado social sobre el cual inciden, pero sobre todo por su carácter imperativo, siendo éste revestido de la autoridad legítima y soberana del poder público"3
Algunos criterios considerados para la formulación de política pública son: la corresponsabilidad, el enfoque de derechos, la solidaridad, la participación, la descentralización y la equidad. La política pública se distingue de la política gubernamental porque esta última obedece a las estrategias de acción diseñadas por un gobierno de turno que actuando en su jurisdicción puede ocuparse de temas que no necesariamente obedecen al genuino interés público. La educación es comprendida como un asunto de interés y de dominio público en clara relación con los procesos de construcción social de la comunidad, por tanto es asunto de la política pública.

Sin embargo, es bien sabido que la maquinaria educacional se materializó en un sistema totalizante que desplazó la soberanía de los Estado-Nación por una soberanía global que circula dentro de una lógica única de dominación. Es decir, fuerzas de poder externas a los mismos Estados han fijado parámetros que rigen las políticas educativas en cada país; así lo afirma Morrow y Torres $(2005,37)$ cuando señalan que la urgencia por expandir las relaciones de mercado ha tenido sus efectos en los sistemas educativos "mientras en el viejo orden capitalista estaba orientado fundamentalmente hacia la formación de una fuerza de trabajo disciplinada y confiable... la nueva economía global requiere de trabajadores con la capacidad de aprender rápidamente y de trabajar en equipo de manera confiable y creativa", lo que condujo a plantear políticas educativas centradas en el concepto de calidad.

2 Sarmiento, Anzola Libardo, (2005), Conferencia Las Políticas Públicas y el Desarrollo Local. Bogotá febrero 4 y 5 citado en: Plan Nacional de Intervención en Discapacidad, Plan de Acción 2005-2007, recuperado el 14 de febrero de 2007, en http://www.discapacidad.gov.co/p_publica/PLAN\%20NACIONAL2005.doc

3 Das Graças, Rúa María. (1997) citada por Molina Gerardo Carlos (2002), en: Modelo de Formación de Políticas y Programas Sociales citada por Carlos Gerardo Molina. Instituto Interamericano para el Desarrollo Social (INDES), recuperado el 2 de febrero de 2007 en http://economia.unmsm.edu.pe/.pdf 


\section{La cuestión de la calidad}

La denominada década perdida de los años ochenta requirió por parte de los Estados el fortalecimiento de la base empresarial para promover el desarrollo y alcanzar niveles internacionales de competitividad. Ello derivó en reformas radicales y en el diseño de nuevas políticas e instituciones para financiar dichos propósitos.

El desarrollo asumido como crecimiento económico implicó que fuera regulado por las leyes del mercado. La nueva tendencia de la administración pública que enfatiza en la descentralización, la eficiencia, la evaluación por resultados, y la competitividad, también marcó el acento en el control de la calidad. La calidad fue entendida como un mecanismo que garantizaría la satisfacción del usuario del servicio, como un modo de ser que daría como resultado el producto deseado. Referirse a la calidad, es utilizar ese concepto como unidad de medida de la excelencia.

Una educación de calidad será, entonces, una educación que cumple con lo que se espera de ella, tanto desde la óptica individual de la persona, como desde la social... se afirma que el rendimiento académico es el indicador más común de la calidad educativa en investigación educativa; es un predictor muy adecuado de la futura productividad económica de los estudiantes y es la medida de la efectividad de las escuelas" (Francesc y Puig: 1998, 164)
La calidad definida por el valor del mercado, es la especificación mínima necesaria para permitir que el producto en cuestión se venda o genere beneficios de todo orden. Es el umbral por debajo del cual, invertir en educación deja de tener sentido porque ya no es posible pretender extraer un beneficio individual o social de esa inversión. La calidad se define, así, desde categorías como eficiencia, eficacia, competitividad, desempeño, competencias, gestión, etc., lo que representa una parte de la estrategia competitiva y supone formas de organización íntimamente ligadas al control. Se demanda que la calidad sea cuantificable y medible y se busca aplicar técnicas cuantitativas a procesos de producción de todo tipo, incluyendo las tareas académicas ${ }^{4}$.

La poca respuesta de los sistemas educativos a las demandas del mercado los señaló como deficientes respecto a la calidad de sus resultados. Se precisaron estudios más sistemáticos de la calidad de la educación con relación a las habilidades que los empresarios requerían. El concepto de calidad se convierte entonces en el punto central de muchas agendas públicas, es así como en la conferencia mundial Educación Para Todos ${ }^{5}$ realizada en 1990, se acordaron planes de acción para satisfacer las necesidades básicas de aprendizaje, paralelamente se asumió la educación no ya como un derecho sino como un servicio, lo que la acercó a la idea de mejoramiento de la calidad concebido ya por la lógica del mercado (calidad del producto, calidad del proceso, calidad definida por el usuario, calidad definida por el valor del mercado).

4 Confróntese: Glazman, N. Raquel, (2001), Evaluación y exclusión en la enseñanza universitaria, Ed. Paidos Mexicana, México, 61, y Apple, Michel, (2002), Educar como Dios Manda, Capítulo 3. Barcelona, Paidos.

5 http://www.oei.es/efa2000jomtien.htm 


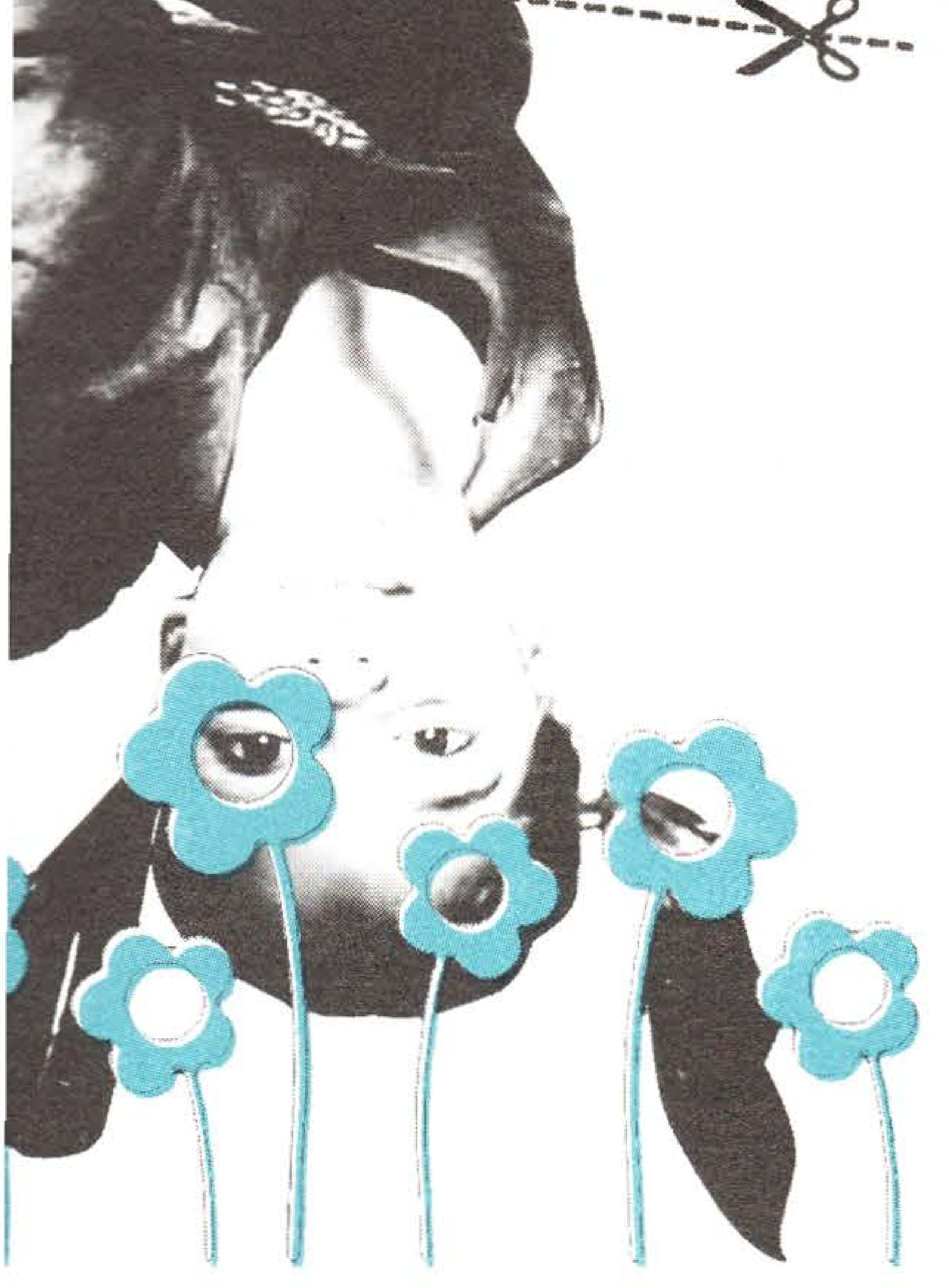

De esta forma se diseñaron indicadores para medir la calidad de la educación y el más visible de ellos fue - y sigue siendo - el del rendimiento escolar, que no sólo mide el nivel de competitividad de los estudiantes sino la efectividad de las instituciones educativas ${ }^{6}$.

Pero el rendimiento escolar no depende tan solo de los procesos internos de la institución y algunos autores ${ }^{7}$ afirman que en realidad depende de aspectos externos como el nivel socioeconómico y el nivel de educación de los padres. La calidad de la educación es determinada por la tradición familiar, el conocimiento enseñado por los maestros y el ambiente social y escolar lo que permitiría inferir que los bajos rendimientos escolares obedecerían más bien a las desventajas sociales de sectores de la población más pobres.

\section{La evaluación de la calidad de la educación:}

La noción de calidad es homologada con los resultados académicos y ello obliga a pensar en sistemas de evaluación que los midan.

La evaluación de la calidad de la educación fue planteada como una necesidad que es satisfecha desde organismos externos a la institución educativa y conformados exclusivamente para tal función. Estos grupos de expertos evaluadores diseñaron estrategias de evaluación que no sólo tienen como fin la regulación y desarrollo del sistema educativo sino primordialmente ejercer control sobre los procesos pedagógicos, verificando que los insumos sean cambiados y adaptados en función de los productos y sobre los procesos administrativos de la Institución educativa, garantizando la rendición de cuentas a la opinión pública

6 Para el caso de Bogotá, ver por ejemplo el estudio Factores asociados al logro académico realizado por Corpoeducación y el Departamento Nacional de Planeación para la Secretaría de Educación en el 2000.

7 Ver el estudio de Pierre Bourdieu sobre capital cultural, escuela y espacio social, así como los trabajos de Peter McLaren, Henry Giroux, Michel Apple y Gimeno Sacristán entre otros. 
Ello crea un círculo perverso en tanto la presión que ejerce la opinión pública sobre los avances del sistema educativo hacen que las evaluaciones se conviertan en un fin en si mismo y no en un medio para valorar procesos pedagógicos y apuntalar a su mejoramiento.

La evaluación tiene entonces por objeto permitir la rendición de cuentas y no verificar que los contenidos y los objetivos de la educación respondan a las necesidades, intereses y capacidades de los alumnos, supeditando entonces la enseñaza, que es adaptada a las exigencias de resultados predeterminados y se preparando a los estudiantes para responder a las evaluaciones y no a los requerimientos de la vida.

El proceso evaluativo se convierte igualmente en un factor de estratificación de la instituciones, de los programas y de las mismas personas que sirve para clasificarlas, catalogarlas y definirlas en una escala que va de excelente a deficiente lo que inevitablemente lleva a la exclusión de los más débiles (los menos competitivos), a la focalización de los recursos en aquellas que han demostrado alcanzar los niveles de excelencia, aumentando cada vez más la inequidad y profundizando la discriminación desde una lógica de castigo y premio, al taylorismo de la educación, buscando la máxima producción al menor costo y al neomarketing, esto es, nuevo mercadeo, de la institución escolar en la que los criterios de calidad se establecen desde la oferta de servicios y productos que atraigan al cliente y en el prestigio social que les ha otorgado la certificación ${ }^{8}$.

La información que se necesita que arroje las evaluaciones responden a la exigencias de quienes financian la educación, por ello la obsolescencia de la investigación educativa radica en su incapacidad de contribuir con datos que reflejen la eficiencia y eficacia de las instituciones educativas. Este tipo de evaluación de la calidad de la educación se basa en modelos estadísticos y en indicadores estandarizados lo que minimiza el valor

8 Algunos colegios están buscando la certificación de calidad ISO 9001 de ICONTEC diseñando sistemas de gestión de calidad para todos sus procesos, a igual que la apropiación de los llamados Modelo Estándar de Control Interno y Modelo de Evaluación centrado en indicadores, que si bien para algunos expertos resultan propicios para "ordenar" la institución es necesario analizar sus repercusiones en las prácticas pedagógicas. Para ver en que consiste la acreditación ver: www.icontec.org, y para ver un ejemplo consultar en educación ver: http://www.estrucplan.com.ar/Producciones/entrega.asp?IDEntrega $=564$ 
social y cultural del conocimiento, el cual vale por que es susceptible de ser evaluado. Las evaluaciones censales, la evaluación por competencias y la evaluación por resultados son instrumentos de control y dominio de la racionalidad instrumental que desarticula la evaluación del universo de la pedagogía.

De otra parte, el nuevo sistema de evaluación escolar desconoce la diversidad de la evaluación según los niveles y ciclos de la educación; homogeniza la aplicación de la norma al conjunto de la educación formal, pública y privada. Desconoce también las variables determinantes del logro académico (condición socioeconómica, medio familiar, oferta educativa, características de los docentes etc.)

En algunos países como Colombia, las pruebas objetivas representan el eje central del sistema de evaluación y acaban por influirlo todo: desde el presupuesto asignado a las instituciones escolares, hasta los contenidos curriculares e incluso los métodos de enseñanza, que tienden a volverse más rígidos pretendidamente en aras de una mayor eficacia. La crítica más severa a este tipo de pruebas es que son totalmente utilitaristas, es decir que presuponen que la educación es algo que se puede utilizar y mesurar.
La evaluación se erige entonces como principal mecanismo para la implementación de la política educativa que trata de organizar la educación de acuerdo con las reglas del mercado. La educación debe ser medida como cualquier otra mercancía: por su valor y por su valor de uso otorgándole así una nueva identidad institucional.

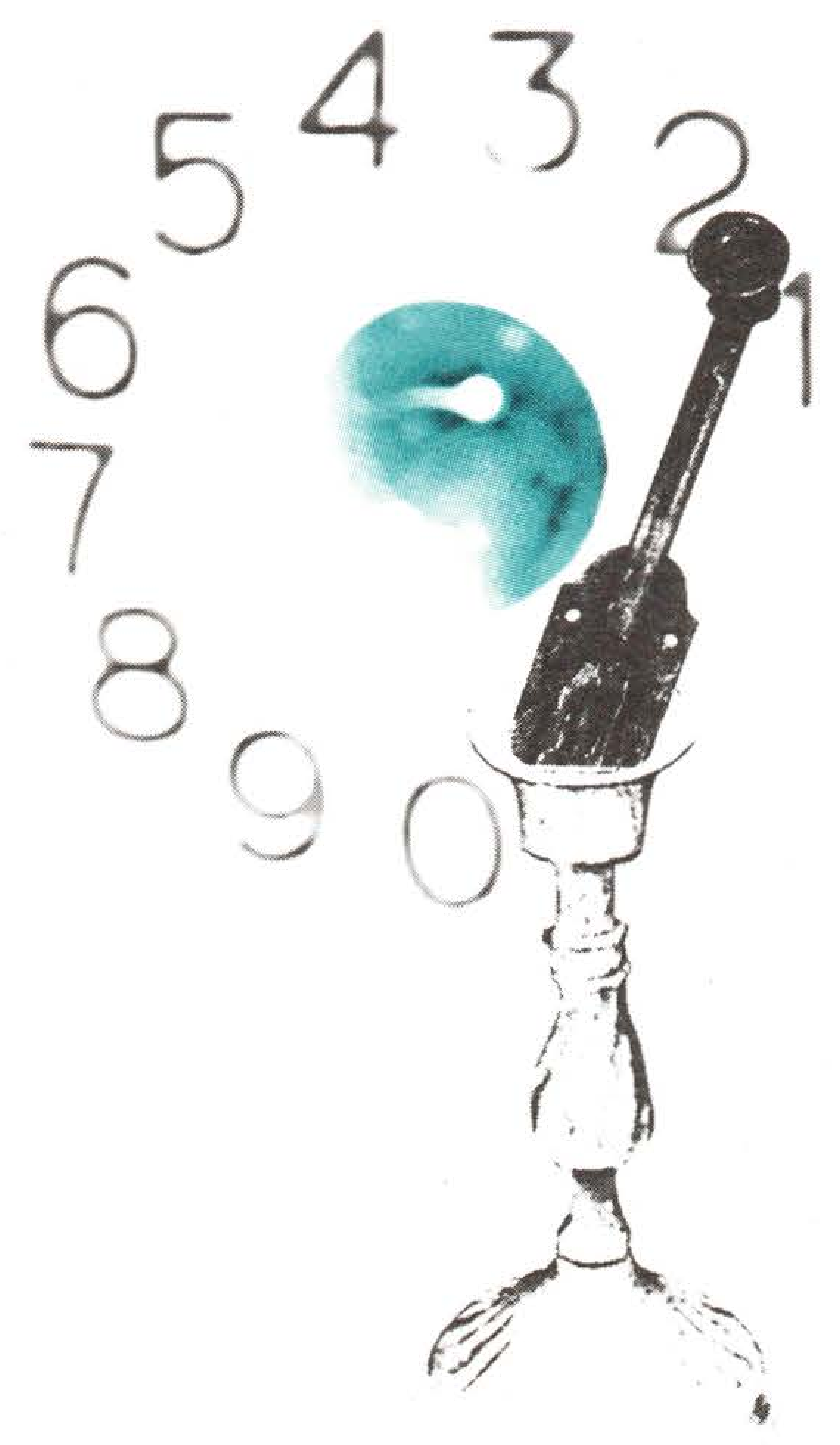




\section{Caracterización de la nueva institución educativa}

Según los criterios de las nuevas políticas educativas, la institución educativa queda convertida en empresa de producción; la autonomía escolar es reemplazada por la autonomía financiera, la financiación gratuita por el autofinanciamiento, la dirección colegiada por la gestión gerencial, la evaluación de procesos por el rendimiento de resultados, los criterios pedagógicos y la formación integral sometidos a la medición de agentes externos.

El discurso de estas políticas se instala en los anuncios de la necesidad de una mayor democracia local y so pretexto de ella se transfieren las responsabilidades, haciendo llamados a la urgencia de un fortalecimiento institucional que la vuelva competente para responder a las demandas de su funciones socializadoras: la vinculación con el mundo del trabajo que es ahora flexible y con el mundo de lo ciudadano que es ahora cosmopolita.

De esta forma nos aproximamos a describir la nueva institucionalidad educativa como:

Una institución eficiente: Entendiendo a la institución educativa como un empresa rentable, que debe generar recursos propios desde un modelo de gestión que proviene de teorías organizacionales y administrativas y que niega la naturaleza académica y pedagógica de la escuela. La institución eficiente es aquella que resuelve sus problemas financieros y logísticos por su propia cuenta pero que responde a políticas centralizadas de evaluación.
Una institución autónoma: El Proyecto Educativo Institucional PEI surge en Latinoamérica como respuesta al agotamiento del modelo de desarrollo educativo, pues este no pudo conciliar crecimiento con calidad. La mirada se dirige así hacia las condiciones de la institución escolar, señalada como el punto crítico del sistema educativo. El quehacer de cada institución, se dijo, deberá estar orientado por su propio proyecto, elaborado de manera autónoma por los actores de cada institución, teniendo en cuanta el contexto de sus estudiantes y las características de su localidad y las intenciones pedagógicas de todos los actores involucrados en la práctica escolar.

Pero el surgimiento de los PEI no estuvo exento de tensiones. La primera de ellas es que si bien el PEI se convirtió en una estrategia para la participación activa de quienes habitan la institución escolar, también lo fue para cambiar el enfoque administrativo del sector, centrando el discurso en la gestión.

La autonomía de las instituciones escolares en Colombia se asoció a las modificaciones de funciones del Ministerio de Educación, dando lugar a estrategias como la de la creación del Sistema Nacional de Evaluación de la Educación orientado a velar por la calidad de la educación. Así, la segunda tensión que se presenta es que la evaluación se centraliza a la par que se otorga autonomía a las Instituciones. 
Sin embargo, como lo afirmaría el profesor Mario Díaz $(2001,11)$,

cuando se habla de autonomía hay que entenderla en términos relativos, pues ella posee unas bases que están reguladas por el poder y el control. Es el Estado el que entonces define la arena o el espacio de autonomía de que puede gozar una institución.. [ ] ...El Estado utiliza la herramienta constitucional para garantizar que la autonomía no desborde los límites establecidos

La autonomía promulgada por la Ley 115 de 1994 ahora se centraba en la autogestión administrativa y autogestión financiera y la evaluación y sus resultados serían primordiales para alcanzar niveles de competitividad en el mercado educativo internacional.

Una institución seudo democrática: Que fomenta la participación de padres, comunidades y docentes en la administración Institucional, en el desarrollo de planes de mejoramiento de la gestión que se reduce a la consecución de recursos, los cuales siendo escasos, enfrentan a la misma comunidad educativa. Es la promoción de una participación para compartir gastos y no para disfrutar de derechos.

De igual manera la democratización del saber se ve vulnerada ante la homogenización, la estandarización del conocimiento y el pensamiento único. La investigación y la innovación pedagógicas son inmovilizadas en tanto estas se perciben como dinamizadoras de organización de colectivos académicos de reflexión, de crítica y de acción transformadora que sin duda configuran comunidades que, como señalara el profesor Juan Carlos Orozco, $(2003,11)$ en su ejercicio de la vigilia intelectual entra a disputar el control ideológico de la educación y el control político del magisterio a las instancias y sectores de la sociedad que, tradicionalmente, lo han sustentado.

Una institución corresponsable: de la cobertura y la calidad, mas no de la evaluación. Así la asignación de recursos esta dada por esos dos criterios y por varios principios entre ellos:

- La financiación compartida

- La asignación de los recursos en función de la eficiencia

- La focalización de los recursos hacia los mas pobres

- Los subsidios a la demanda

La garantía de cobertura en relación con la calidad (tal y como se ha definido en este documento) les permite a las instituciones con mas prestigio establecer criterios de selección que acentúan la discriminación y la inequidad en el acceso a las oportunidades de las poblaciones mas pobres y en relación con la asignación del presupuesto obliga a las mas desacreditadas (que por lo general son las oficiales) a admitir mayor número de estudiantes (capitación) sin tener las condiciones para atenderlos.

Una institución eficaz y efectiva: En tanto adapta los PEI, programas y los currículos a las exigencias de los desarrollos tecnológicos, las competencias básicas, las competencias laborales y las competencias ciudadanas que garanticen una persona formada para el mundo del mercado como productor pero también como consumidor. 


\section{A modo de conclusión}

Las políticas educativas de los últimos tiempos se hallan lejos de configurarse como políticas públicas en la medida en que los intereses de un solo sector, el económico han logrado tener primacía sobre los demás. En palabras de Torres $(2005,56)$ "la posición debilitada del Estado, a su vez ha abierto camino para el incremento de la educación, asî como para las reclamaciones de una reforma de la educación en respuesta a los supuestos imperativos de la globalización”.

Enunciar como propósito de una auténtica política pública la calidad de la educación, exigirá grandes esfuerzos por deconstruir toda esta carga semántica de lo que ha significado el término calidad en educación y llenarlo de nuevos sentidos que se reflejen en acciones que hagan visibles factores como el trabajo intersectorial con las familias, políticas de formación docente que incentiven la investigación en el aula, y fortalezcan experiencias pedagógicas trasformadoras.
Esto sin duda también hace una invitación a los investigadores de la educación en tanto se vislumbran nuevos objetos de análisis en el campo de los estudios comparados que para el caso colombiano pueden situar en los últimos años a Medellín y a Bogotá como experiencias de política pública educativa alternativas y cuyos sentidos y efectos están por considerarse.

Finalmente, no habrá" que desestimar la idea de que algunos maestros y maestras en zonas recónditas de este país y aún en las metropolitanas hayan re - creado estos discursos para constituir líneas de fuga a los imperativos de la calidad y hayan producido nuevos sentidos de lo que significa una buena educación, una educación digna en contextos la mayor de las veces indignos. Maestros y maestras que hayan creado experiencias pedagógicas que subviertan el significado hegemónico de calidad sin que el ojo del gran hermano lo haya detectado aún. 


\section{Bibliografía}

Apple, M. Educar como Dios Manda. Capítulo 3. Barcelona: Paidós. Compilación de Autores Varios. (2002) Estándares educativos. Evaluación y Calidad de la educación. Bogotá, D. C.: Cooperativa Editorial Magisterio. 1 ${ }^{\mathrm{a}}$. ed., 2002.

Bárcena, Fernando. El oficio de la ciudadanía. Barcelona: Paidós, 1995.

Das Graças, Rúa María. Citada por Molina Gerardo Carlos (2002), en: Modelo de Formación de Políticas y Programas Sociales. Instituto Interamericano para el Desarrollo Social (INDES), [en línea]: en http://economia.unmsm. edu.pe/.pdf, registrado el 2 de febrero de 2007.

Díaz, Mario, otros. La Autonomía escolar: alcances y contrapuntos, en: Revista Educación y Cultura No. 57, CEID - FECODE. Bogotá, D.C., 2001.

Frances, Pedro y Puig, Irene. Las reformas educativas. Una perspectiva política y comparada, Capítulo 2.

Barcelona: Ediciones Paidos Ibérica, S.A., 1998.

Giroux, Henry. Cultura política y práctica educativa. Barcelona: Grao, 2001.

Glazman, N. Raquel. Evaluación y exclusión en la enseñanza universitaria. México: Editorial Paidós, 2001.

Libreros, Daniel y Otros. Tensiones de las políticas educativas en Colombia. Bogotá, D.C.: Universidad Pedagógica Nacional, 2002.

Morrow Raymond, Torres Carlos Alberto. Estado, Globalización y Política Educacional, en: Burbules, 2005. Nicholas Torres, Carlos Alberto (Coord.) Globalización y Educación. Madrid: Editorial Popular.

Orozco, Juan Carlos. Gestión educativa. Nueva institucionalidad, Módulo. Bogotá, D.C.: CINDE, 2005.
Reyes, F. Gobierno escolar y organización estudiantil. Bogotá, D.C.: Alcaldía Mayor - Publicaciones, Universidad Distrital, 2002.

Sarmiento, Anzola Libardo, (2005), Conferencia Las Políticas Públicas y el Desarrollo Local. Bogotá febrero 4 y 5, en: Plan Nacional de Intervención en Discapacidad, Plan de Acción 2005 - 2007 consultado el 14 de febrero de 2007, en http://www.discapacidad.gov.co/p_publica/ PLAN\%20NACIONAL2005.doc

SED - IDEP, Documento interno de trabajo preparatorio del Foro Educativo Distrital, Bogotá, D.C., 2004. 